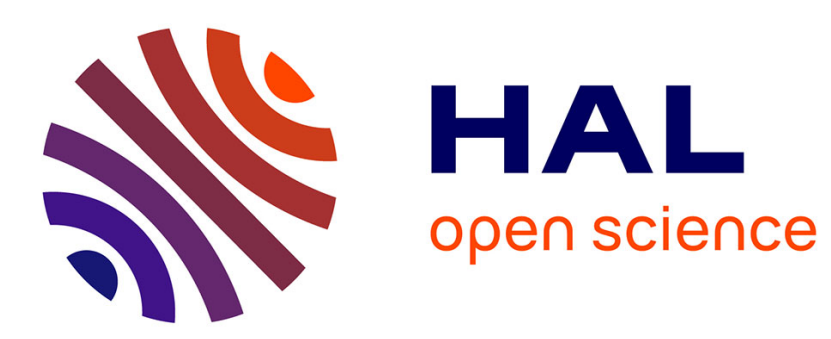

\title{
A single landmark based localization algorithm for non-holonomic mobile robots
}

Hugues Sert, Annemarie Kokosy, Wilfrid Perruquetti

\section{To cite this version:}

Hugues Sert, Annemarie Kokosy, Wilfrid Perruquetti. A single landmark based localization algorithm for non-holonomic mobile robots. IEEE International Conference on Robotics and Automation, May 2011, Shanghai, China. inria-00602536

\section{HAL Id: inria-00602536 https://hal.inria.fr/inria-00602536}

Submitted on 23 Jun 2011

HAL is a multi-disciplinary open access archive for the deposit and dissemination of scientific research documents, whether they are published or not. The documents may come from teaching and research institutions in France or abroad, or from public or private research centers.
L'archive ouverte pluridisciplinaire HAL, est destinée au dépôt et à la diffusion de documents scientifiques de niveau recherche, publiés ou non, émanant des établissements d'enseignement et de recherche français ou étrangers, des laboratoires publics ou privés. 


\title{
A single landmark based localization algorithm for non-holonomic mobile robots
}

\author{
Hugues Sert, Annemarie Kökösy and Wilfrid Perruquetti
}

\begin{abstract}
This paper proposes a single landmark based localization algorithm for non-holonomic mobile robots. In the case of a unicycle robot model, the localization problem is equivalent to the system observability. Based on this observation, the proposed localization method consists in finding a vector function which depends on the measurement vector and its derivatives. In order to compute estimates of the successive derivatives of the measurement vector, we will use a numerical differentiation method. When the robot is able to only measure the relative angle between itself and the landmark in $2 \mathrm{D}$ case, the algorithm estimates the posture of the robot, under the hypothesis that control inputs are known. But, sometimes it is also useful to be able to estimate the control input (for example when the robot slips). This is possible with the proposed algorithm by using a landmark in dimension three. The simulation results will be given in order to show the effectiveness of the proposed algorithm. Moreover, these results are compared with those obtained by an Extended Kalman Filter in order to underline the advantages of the new algorithm.
\end{abstract}

\section{INTRODUCTION}

Localization is one of the most important issues for mobile entity autonomous navigation [1]. Indeed it is impossible to look for an intelligent mobile robot navigation strategy if the robot has no self-localization capabilities. This localization problem has focused researchers' efforts for a long time. It admits two sub-classes:

1) absolute localization: the collected data allow to localize the robot in the global environment (using for example GPS),

2) relative localization: the collected data allow to localize the robot with respect to the current situation (using for example odometers or other proprioceptive sensors).

In an indoor environment it is almost very difficult to get absolute localization because it is impossible to use GPS (satellite signals are no more efficient indoors). Odometers or similar proprioceptive sensors have an important drawback because of drift issues [2]. Thus, in order to solve the localization problem, the researchers have developed landmarks based localization methods: landmarks are points of known position in the environment which can be "seen" by the

Annemarie Kökösy and Hugues Sert are with ISEN and LAGIS FRE CNRS 3303, 41 bvd Vauban, 59046 Lille Cedex, France (e-mail: annemarie.kokosy@isen.fr, hugues.sert@isen.fr).

Wilfrid Perruquetti is with ALIEN INRIA - LNE, Parc Scientifique de la Haute Borne 40, avenue Halley Bt.A, Park Plaza 59650 Villeneuve d'Ascq and LAGIS FRE CNRS 3303, Ecole Centrale de Lille, BP 48, Cité Scientifique, 59651 Villeneuve-d'Ascq, France (e-mail: wilfrid.perruquetti@inria.fr).

This work was partially supported by ALIEN INRIA - LNE, Mission Insertion Thales and the INTERREG IV A 2 Mers Seas Zeen Cross-border Cooperation Program 2007-2013 under SYSIASS 6-20 project. robot. Then the localization problem can be formulated as an observability problem (in the sense of the automatic control community): see [3], [4] and [5] for one or more robots. One main drawback of such obtained results is that the system is observable only for more than three landmarks. In some environment it could be difficult to find so much landmarks (see for example [6]), so other authors have developed single landmark based methods which require to compute a scale factor. [7] and [8] use the shapes of the landmarks which require to use artificial landmarks of known position and shape. If the shapes of the landmarks are not known, it is possible to use information about the robot movement in order to estimate the scale factor (see [6]). [9] uses the odometers to estimate the scale factor. The greatest drawback of this method is the robustness. In this paper a new localization method is proposed which uses only the position of the landmarks and the relative angle between the landmarks and the robot to estimate, the linear speed, the angular speed and the position of the robot with only one landmark.

\section{Problem formulation of A NEW Single LANDMARK BASED LOCALIZATION}

\section{A. Notations}

In this paper the following notations are used (see Fig. 1): - $P=[x, y, \theta]$ is the robot posture which contains the coordinates $[x, y]$ of the robot and its orientation $\theta$,

- $\left[x_{A i}, y_{A i}, z_{A i}\right]$ is the coordinates of the landmark,

- the relative coordinates robot-landmarks:

$$
x_{r}=x_{A i}-x, \quad y_{r}=y_{A i}-y,
$$

- $\alpha$ and $\beta$ the relative angles robot-landmarks $A_{i}$,

- the distance between the robot and the landmark:

$$
\text { - } d_{R A i}=\sqrt{x_{r}^{2}+y_{r}^{2}+z_{A i}^{2}} \text { in space, }
$$$$
\text { - } d_{R A i s o l}=\sqrt{x_{r}^{2}+y_{r}^{2}} \text { in plane, }
$$

- for a physical variable $v, v_{m}$ will denote its measured quantity (usually $v_{m}=v+\eta_{v}$ where $\eta_{v}$ is an additive noise) and $v_{f}$ will denote a filtered value of the measured quantity $v_{m}$ which should be quite similar to $v$ in the ideal case.

- $\hat{x}$ will denote the estimate value of variable $x$.

\section{B. Problem}

Let

$$
\left\{\begin{array}{l}
\dot{X}=F(X, V) \\
Z=H(X)
\end{array}\right.
$$




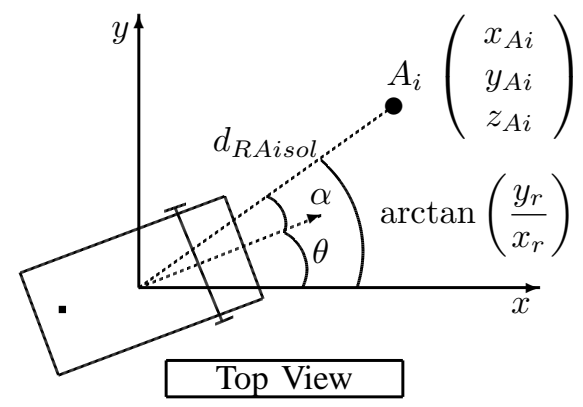

- $A_{i}$ : Landmark $A_{i}$

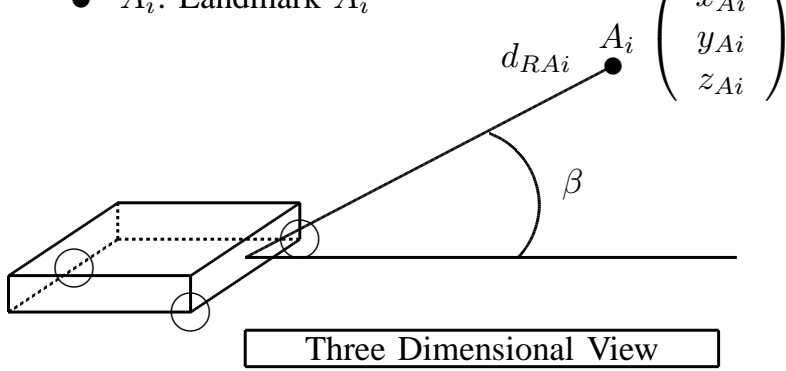

Fig. 1. Robot and landmark notation for the localization

be a state model system of a mobile robot, where $X \in \mathbb{R}^{n}$ is the state vector containing the mobile robot posture $P=$ $[x, y, \theta], V \in \mathbb{R}^{m}$ is the control input vector and $Z \in \mathbb{R}^{p}$ is the measurement vector. Since localization consists in finding the posture of the robot (which is a part of the state vector) from the measured output, it is clear that such a problem is closely linked to the observability problem (in fact it is a partial observability problem). Such a problem has two main points of view: a differential geometric one and an algebraic one. Let us recall here, one of the most important results obtained by Michel Fliess about observability in an algebraic framework:

Theorem 1 (see [10] and [11]): A system variable $x \in F$ is said to be observable if, and only if, it is algebraic over $f\langle u, y\rangle$. An input-output system $F / f$ is said to be observable if, and only if, the extension $F / f\langle u, y\rangle$ is algebraic.

Where the following definitions are used: for $F$ which is a differential field, the differential field extension $L / F$ is given by two differential fields $F, L$, such that the derivation of $F \subset L$ is the restriction to $F$ of the derivation of $L$. An element of $L$ is said to be differentially algebraic over $F$ if, and only if, it satisfies an algebraic differential equation with coefficients in $F . F\langle S\rangle$, where $S$ is a subset of $L$, the differential subfield of $L$ generated by $F$ and $S$.

Thus the above mentioned result shows that a system variable $x \in F$ is said to be observable, if and only if there is an algebraic equation linking $x$, the outputs, the inputs and a finite number of the time derivatives of the inputs and outputs. Let's use this algebraic criteria for our localization problem.

Theorem 2: A mobile robot with state dynamics (2) is said to be localizable if, and only if, there is an algebraic equation linking posture $P$, the measured output $Z$, input $V$ and a finite number of the time derivatives of $Z$ and $V$.

Thus, based on this result, our proposed method of localization consists in finding the vector function $K\left(Z, \dot{Z}, \ddot{Z}, \ldots, Z^{(q)}\right)$ such that:

$$
P=K\left(Z, \dot{Z}, \ddot{Z}, \ldots, Z^{(q)}\right) .
$$

Where $Z^{(q)}$ is the $q$-derivative of $Z$.

For our case study, a unicyle robot, whose posture is exactly state $X$, thus its localizability is equivalent to the system observability (see [10] and [12] for additional results on the observability of non linear systems). The knowledge of the formal expression of $K$ and an estimation of the successive derivatives of $Z$, will lead to the reconstruction of posture $P$ for any time $t$ : this is the point of view used in this paper which is closely linked to the new algorithms developed within the ALIEN - INRIA project for numerical differentiation estimation (see [13]). Let us emphasize that these methods, which are algebraic and non-asymptotic, exhibit good robustness properties with respect to corrupting noise, without the need of knowing their statistical properties. To sum up, the localization (reconstruction of $P=X$ (for our case study)) is then obtained through three steps:

1) get a formal expression of $K$ (see theorems 3 and 4),

2) compute the estimates of the successive derivatives of $Z$ (see sub-section II-E) (for this case study, there is no need to estimate the derivatives of $V$ ),

3 ) in $K$, substitute the derivatives by their estimates.

\section{Robot State Model}

The mobile robot considered is of unicycle type, with two driving wheels mounted on the same axis and independently controlled by two actuators (DC motors). The robot is fully described by a three dimensional vector of generalized coordinates $X$ constituted by the coordinates $[x, y]$ of the midpoint between the two driving wheels and by the orientation angle $\theta$ with respect to a fixed frame. This non-holonomic robot is assumed to observe landmarks in two or three dimensions (both cases will be dealt with). The kinematic model of this type of robot is given by:

$$
\dot{X}=F(X, V)=\left[\begin{array}{c}
u \cos (\theta) \\
u \sin (\theta) \\
\omega
\end{array}\right]
$$

where $X=\left[\begin{array}{ccc}x & y & \theta\end{array}\right]^{T}$ is the posture of the robot and $V=\left[\begin{array}{ll}u & \omega\end{array}\right]^{T}$ is the control input (linear velocity, angular velocity).

\section{Measurement Model}

We are going to distinguish the $2 \mathrm{D}$ and $3 \mathrm{D}$ cases. The measurement model

- in the $2 \mathrm{D}$ case, is given by equations (5) and (6):

$$
\begin{aligned}
Z & =H(X)=\alpha_{m}=\alpha+\eta_{\alpha} \\
\alpha & =\arctan \left(\frac{y_{r}}{x_{r}}\right)-\theta
\end{aligned}
$$


where $x_{r}$ and $y_{r}$ are the relatives positions between the landmarks and the robot defined by (1) and $\eta_{\alpha}$ is a measurement noise.

- in the 3D case, is given by equations (7)-(9):

$$
\begin{aligned}
Z & =H(X)=\left[\begin{array}{c}
\alpha_{m}=\alpha+\eta_{\alpha} \\
\beta_{m}=\beta+\eta_{\beta}
\end{array}\right] \\
\alpha & =\arctan \left(\frac{y_{r}}{x_{r}}\right)-\theta \\
\beta & =\arctan \left(\frac{z_{A i}}{\sqrt{x_{A i}^{2}+y_{A i}^{2}}}\right)
\end{aligned}
$$

where $\eta_{\alpha}$ and $\eta_{\beta}$ are additive noise measurements.

\section{E. Estimation of the successive time derivatives of $Z$}

This algebraic setting for numerical differentiation of noisy signals was introduced in [14] and analyzed in [13]. Consider a signal $v_{m}=v+\eta_{v}$. We want to estimate the derivative of $v$.

1) Continuous version of the derivative: The continuous version of the $n^{\text {th }}$ time derivative estimate of the variable $v$ is given by:

$$
v_{f}^{(n)}(T t ; \kappa ; \mu ; N)=\int_{0}^{1} g(\tau, \kappa, \mu, N) v_{m}(t-\tau) d \tau
$$

where $v_{m}$ is the measured quantity of $v$ (see notations) and

$$
g(\tau)=\sum_{l=0}^{q} \lambda_{l} h_{\kappa+q-l, \mu+l}(\tau),(\kappa ; \mu) \in \mathbb{N}, q=N-n
$$

with

$$
\lambda_{l}=(-1)^{q-l}\left(\begin{array}{c}
p+q-l \\
p
\end{array}\right)\left(\begin{array}{c}
p+q+1 \\
l
\end{array}\right), l=0, \ldots, q
$$

where $q=N-n$ and $p=n+\kappa$ and

$$
\begin{aligned}
h_{\kappa, \mu}(\tau) & =\frac{(-1)^{n} \gamma_{\kappa, \mu, n}}{T^{n}} \operatorname{rect}(\tau) \frac{d^{n}}{d \tau^{n}} \omega_{\kappa, \mu}(\tau) \\
\operatorname{rect}(\tau) & =\left\{\begin{array}{c}
1 \text { if } \tau \in[0 ; 1] \\
0 \text { ortherwise }
\end{array}\right. \\
\omega_{\kappa, \mu}(t) & =t^{\kappa+n}(1-t)^{\mu+n} \\
\gamma_{\kappa, \mu, n} & =\frac{(\mu+\kappa+2 n+1) !}{(\mu+n) !(\kappa+n) !}
\end{aligned}
$$

See [13] for details about the choice of the different parameters involved in this differentiation estimation method.

Remark 1: Let us note that this formula is still valid for $n=0$ and thus gives a filtered estimate of the measured variable. This will be used to obtain the following filtered quantities: $\alpha_{f}, \beta_{f}, u_{f}, \omega_{f}, \theta_{f}, \ldots$

2) Discrete version: The $n^{\text {th }}$ order time derivative estimate in the discrete case is obtained as the output of the finite impulse response filter (FIR):

$$
v^{(n)}\left(l T_{s} ; \kappa ; \mu ; N\right) \approx \sum_{j=0}^{M} W_{j} g_{j} v_{m, l-j}
$$

where $v_{m, i}=v_{m}\left(i T_{s}\right), T_{s}$ is the sampling period, $M$ is the number of coefficients of the filter and $c_{j}=W_{j} g_{j}, j=$ $0, \ldots, M-1$ is its impulse response. Coefficients $W_{j}$ correspond to the chosen method of integration of the signal between 0 and 1 . Here, for the trapezoidal method, the coefficients are given by:

$$
\begin{gathered}
W_{0}=W_{M}=\frac{1}{2 M} \\
W_{j}=\frac{1}{M}, j=1, \ldots, M-1
\end{gathered}
$$

Coefficients $g_{j}$ are such as $g_{j}=g\left(j T_{s} / T\right), j=0, \ldots, M$, with $T=M T_{s}$ the first moment of estimation.

\section{MAIN RESULTS}

\section{A. New $2 D$ and $3 D$ robot localization algorithms}

1) $2 D$ case: A new single landmark based localization algorithm:

Hypothesis 1: The following data are supposed to be known:

- $\left[x_{A i}, y_{A i}, z_{A i}\right]$, the landmark position,

- $u$, the linear speed of the robot,

- $\omega$, the angular speed of the robot.

The following data are supposed to be measured:

- $\theta_{m}$, measured with a compass (a noisy measurement),

- $\alpha_{m}$ the robot-landmark relative angle (a noisy measurement).

Theorem 3: Let us consider the robot state model, (4), the measurement model, (5) and (6) and the relative coordinates between the robot and the landmark $x_{r}$ and $y_{r}$ given by (1). Under hypothesis 1 , the relative coordinates of the robot, $X_{r}=\left[x_{r}, y_{r}\right]^{T}$ are estimated by:

$$
\hat{X}_{r}=\left[\begin{array}{c}
\hat{x}_{r} \\
\hat{y}_{r}
\end{array}\right]=\left[\begin{array}{c}
\frac{u_{f} \sin \left(\alpha_{f}\right) \cos \left(\alpha_{f}+\theta_{f}\right)}{\left(\dot{\alpha}_{f}+\omega_{f}\right)} \\
\frac{u_{f} \sin \left(\alpha_{f}\right) \sin \left(\alpha_{f}+\theta_{f}\right)}{\left(\dot{\alpha}_{f}+\omega_{f}\right)}
\end{array}\right]
$$

where $u_{f}, \omega_{f}, \alpha_{f}, \dot{\alpha}_{f}$ and $\theta_{f}$ are the filtered quantities (see remark 1).

Remark 2: This algorithm is suitable only when the nonholonomic constraint is satisfied (no slipping, no skidding). More over the following condition should be in force $\dot{\alpha}+\omega \neq$ 0 : for example when the landmarks is far away from the robot $\dot{\alpha} \simeq 0$ and the robot is not spinning (in line) $\omega \simeq 0$ or when the robot is at rest.

Proof: By taking the time derivative of (1) combined with (4), the following equation is obtained:

$$
\dot{x}_{r}=-\dot{x}=-u \cos (\theta) \text { and } \dot{y}_{r}=-\dot{y}=-u \sin (\theta) .
$$

By using equation (6), after the filtering process (i.e. removing the noise effect $\eta$ ):

$$
\sin \left(\alpha_{f}+\theta\right) \hat{x}_{r}-\cos \left(\alpha_{f}+\theta\right) \hat{y}_{r}=0
$$

The time derivative of this equation combined with (20), leads to:

$$
\begin{aligned}
& \left(\dot{\alpha}_{f}+\omega\right) \cos \left(\alpha_{f}+\theta\right) \hat{x}_{r}-\sin \left(\alpha_{f}+\theta\right) u \cos (\theta)= \\
& -\left(\dot{\alpha}_{f}+\omega\right) \sin \left(\alpha_{f}+\theta\right) \hat{y}_{r}-\cos \left(\alpha_{f}+\theta\right) u \sin (\theta)
\end{aligned}
$$


$\left(\dot{\alpha}_{f}+\omega\right)\left[\cos \left(\alpha_{f}+\theta\right) \hat{x}_{r}+\sin \left(\alpha_{f}+\theta\right) \hat{y}_{r}\right]=$

$\sin \left(\alpha_{f}+\theta\right) u \cos (\theta)-\cos \left(\alpha_{f}+\theta\right) u \sin (\theta)$

So:

$$
\left(\dot{\alpha}_{f}+\omega\right)\left[\cos \left(\alpha_{f}+\theta\right) \hat{x}_{r}+\sin \left(\alpha_{f}+\theta\right) \hat{y}_{r}\right]=u \sin \left(\alpha_{f}\right)
$$

Now (21) and (24) can be rewritten into a matrix form with $\hat{x}_{r}$ and $\hat{y}_{r}$ as unknown data:

$$
\begin{gathered}
{\left[\begin{array}{cc}
\sin \left(\alpha_{f}+\theta\right) & -\cos \left(\alpha_{f}+\theta\right) \\
\left(\dot{\alpha}_{f}+\omega\right) \cos \left(\alpha_{f}+\theta\right) & \left(\dot{\alpha}_{f}+\omega\right) \sin \left(\alpha_{f}+\theta\right)
\end{array}\right]\left[\begin{array}{l}
\hat{x}_{r} \\
\hat{y}_{r}
\end{array}\right]=} \\
{\left[\begin{array}{c}
0 \\
u \sin \left(\alpha_{f}\right)
\end{array}\right]}
\end{gathered}
$$

Solving this equation it follows that:

$$
\hat{X}_{r}=\left[\begin{array}{c}
\hat{x}_{r} \\
\hat{y}_{r}
\end{array}\right]=\left[\begin{array}{c}
\frac{u \sin \left(\alpha_{f}\right) \cos \left(\alpha_{f}+\theta\right)}{\left(\dot{\alpha}_{f}+\omega\right)} \\
\frac{u \sin \left(\alpha_{f}\right) \sin \left(\alpha_{f}+\theta\right)}{\left(\dot{\alpha}_{f}+\omega\right)}
\end{array}\right] .
$$

Then $u, \omega$ and $\theta$ are also filtered in order to increase the robustness of the estimation process with respect to the noise which could affect the control inputs and measurements.

Remark 3: We can remark in hypothesis 1 that, in order to estimate $\hat{X}_{r}$ by using a 2D landmark, it is necessary to know the linear and angular speed of the robot. If this hypothesis is too restrictive, it can be relaxed by using a three-dimensional landmark. In this case, it is possible to estimate the linear and the angular speed of the robot.

2) 3D case: A new single landmark based localization algorithm without the knowledge of the linear and angular speed: Contrary to hypothesis 1 in the 2D case, when a 3D landmark is used, it is not necessary to know the linear and angular speeds of the robot.

Hypothesis 2: The following features are assumed to hold:

- landmark position $\left[x_{A i}, y_{A i}, z_{A i}\right]$ is known,

- relative angles $\alpha_{m}$ and $\beta_{m}$ are measured (noisy measurement),

- $\theta_{m}$, the robot orientation is measured with a compass (a noisy measurement).

Theorem 4: Let us consider the robot state model, (4), the measurement model, (7)-(9) and the relative coordinates between the robot and the landmark $x_{r}$ and $y_{r}$ given by equation (1). Under hypothesis 2 the relative coordinates of the robot, $X_{r}=\left[x_{r}, y_{r}\right]^{T}$ and the velocities (linear $u$ and angular $\omega$ ) are estimated by:

$$
\begin{aligned}
\hat{u} & =\frac{z_{A i} \dot{\beta}_{f}}{\sin ^{2} \beta_{f} \cos \alpha_{f}} \\
\hat{\omega} & =\frac{2 \tan \left(\alpha_{f}\right)}{\sin \left(2 \beta_{f}\right)} \dot{\beta}_{f}-\dot{\alpha}_{f} \\
\hat{X}_{r} & =\left[\begin{array}{c}
\hat{x}_{r} \\
\hat{y}_{r}
\end{array}\right]=\left[\begin{array}{c}
\frac{\hat{u} \sin \left(\alpha_{f}\right) \cos \left(\alpha_{f}+\theta_{f}\right)}{\left(\dot{\alpha}_{f}+\hat{\omega}\right)} \\
\frac{\hat{u} \sin \left(\alpha_{f}\right) \sin \left(\alpha_{f}+\theta_{f}\right)}{\left(\dot{\alpha}_{f}+\hat{\omega}\right)}
\end{array}\right]
\end{aligned}
$$

where $\alpha_{f}, \dot{\alpha}_{f}, \beta_{f}, \dot{\beta}_{f}$ and $\theta_{f}$ are the filtered quantities (see remark 1).
Proof: The only part to be proved is the estimation of the velocities (for the proof of equation (27) see the 2D case).

\section{Estimation of the linear speed $u$}

Using Fig. 1:

$$
\left[\begin{array}{l}
x_{r} \\
y_{r}
\end{array}\right]=d_{\text {RAisol }}\left[\begin{array}{c}
\cos (\alpha+\theta) \\
\sin (\alpha+\theta)
\end{array}\right]
$$

Using the filtering process to eliminate the noise measurement, this equation becomes:

$$
\left[\begin{array}{l}
\hat{x}_{r} \\
\hat{y}_{r}
\end{array}\right]=d_{\text {RAisol }}\left[\begin{array}{c}
\cos \left(\alpha_{f}+\theta_{f}\right) \\
\sin \left(\alpha_{f}+\theta_{f}\right)
\end{array}\right]
$$

So:

$$
d_{R A i s o l}=\hat{x}_{r} \cos \left(\alpha_{f}+\theta_{f}\right)+\hat{y}_{r} \sin \left(\alpha_{f}+\theta_{f}\right)
$$

By differentiating this equation and by using equation (20), the following equation is obtained:

$$
\begin{gathered}
\dot{d}_{R \text { Aisol }}=-\hat{x}_{r}\left(\dot{\alpha}_{f}+\hat{\omega}\right) \sin \left(\alpha_{f}+\theta_{f}\right) \\
-\hat{u} \cos \theta_{f} \cos \left(\alpha_{f}+\theta_{f}\right) \\
+\hat{y}_{r}\left(\dot{\alpha}_{f}+\omega_{f}\right) \cos \left(\alpha_{f}+\theta_{f}\right)-\hat{u} \sin \theta_{f} \sin \left(\alpha_{f}+\theta_{f}\right)
\end{gathered}
$$

which combined with equation (21) leads to:

$$
\dot{d}_{R A i s o l}=-\hat{u} \cos \left(\alpha_{f}\right) \text {. }
$$

Using equation (9):

$$
\tan \beta_{f}=\frac{z_{A i}}{d_{R A i s o l}}
$$

which can be rewritten in the following expression:

$$
d_{R A i s o l} \sin \beta_{f}-z_{A i} \cos \beta_{f}=0 .
$$

The following equation can be obtained by differentiating this last one:

$$
\dot{d}_{R A i s o l} \sin \beta_{f}+\dot{\beta}_{f} d_{R A i s o l} \cos \beta_{f}+z_{A i} \dot{\beta}_{f} \sin \beta_{f}=0 .
$$

In this relation, by replacing $\dot{d}_{R A i s o l}$ by equation (29):

$$
-\hat{u} \cos \alpha_{f} \sin \beta_{f}+\dot{\beta}_{f}\left(d_{R A i s o l} \cos \beta_{f}+z_{A i} \sin \beta_{f}\right)=0 .
$$

And by using Fig. 1:

$$
\left[\begin{array}{c}
d_{R A i s o l} \\
z_{A i}
\end{array}\right]=d_{R A i}\left[\begin{array}{c}
\cos \beta_{f} \\
\sin \beta_{f}
\end{array}\right]
$$

so:

$$
d_{R A i}=d_{R A i s o l} \cos \beta_{f}+d_{R A i s o l} \sin \beta_{f}
$$

By replacing this equation in (31) and by knowing that $z_{A i}=$ $d_{R A i} \sin \beta_{f}$, (Fig. 1):

$$
\hat{u}=\frac{\dot{\beta}_{f} d_{R A i}}{\cos \alpha_{f} \sin \beta_{f}}=\frac{\dot{\beta}_{f} z_{A i}}{\cos \alpha_{f} \sin ^{2} \beta_{f}}
$$

\section{Estimation of the angular speed $\omega$}

By using the expression of $d_{R A i s o l}$ found in (28) in (24):

$$
\left(\dot{\alpha}_{f}+\hat{\omega}\right) d_{R A i s o l}=\hat{u} \sin \alpha_{f}
$$

So by using equation (9):

$$
\hat{\omega}=\frac{\hat{u} \sin \alpha_{f} \tan \beta_{f}}{z_{A i}}-\dot{\alpha}_{f}
$$


By replacing $\hat{u}$ found in equation (33), the result for $\hat{\omega}$ is demonstrated:

$$
\hat{\omega}=\frac{\dot{\beta}_{f} \tan \alpha_{f}}{\sin \beta_{f} \cos \beta_{f}}-\dot{\alpha}_{f}=\frac{2 \dot{\beta}_{f} \tan \alpha_{f}}{\sin \left(2 \beta_{f}\right)}-\dot{\alpha}_{f}
$$

\section{B. Experimental results and comparisons with an extended Kalman filter}

In order to show the effectiveness of the here proposed method, the obtained results from theorem 4 were implemented on Matlab (hereafter called ALIEN algorithm). Results from theorem 3 are not implement as they are subparts of theorem 4. Results obtained using ALIEN algorithm are compared with those obtained by an EKF (Extended Kalman Filter) for two parameters setting. In the first one, the statistical noise characteristics ( $R$ matrix) are known by the EKF and in the second one they are unknown (the covariance noise matrix ( $R$ matrix) is so set to high values). In both cases the new algorithm has no information about the noise characteristics, and EKF is initialized at $10 \mathrm{~cm}$ of the true initial position. Due to paper limitation the EKF is not developed here (for more details see [15]).

Tab. I summaries the required inputs (informations) for each algorithm (ALIEN and EKF).

\begin{tabular}{|c|c|c|}
\hline The algorithm : & ALIEN Algorithm & EKF \\
\hline Needs to know the control input & NO & YES \\
Needs to know the noise characteristics & NO & YES \\
Needs to know the orientation of the robot & YES & NO \\
Needs to be initialized & NO & YES \\
\hline
\end{tabular}

TABLE I

COMPARISON OF TWO LOCALIZATION ALGORITHMS HYPOTHESIS

Fig. 2 shows the inputs and outputs for each algorithms. One can see that the ALIEN algorithm only needs the
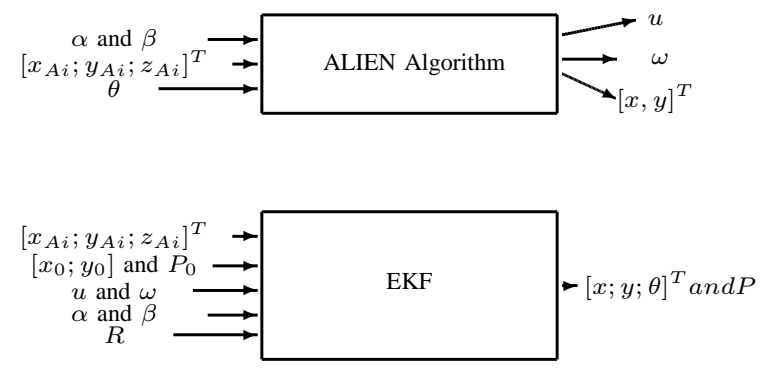

Fig. 2. Input and output of the two localization methods

measurements of $\alpha$ and $\beta$, the landmarks position and the orientation of the robot to get an estimation of the velocities and of the position of the robot contrary to the EKF which needs further noise characteristics (matrix $R$ ), and an initialization $\left(P_{0}\right)$. Furthermore, EKF needs the velocities input contrary to the ALIEN algorithm which does not need this information, as it is able to estimate it. Moreover, the ALIEN algorithm only uses information about a single landmark to get an estimation of the robot velocities and position contrary to the EKF which needs at least three landmarks to get an estimation of the position of the robot. In the case of more than one landmarks, the ALIEN algorithm uses a mathematical means to fuse the different estimations obtained by using each landmark separately.

1) Comparative results: The algorithms are tested for 1 , 5, 10, 50 and 100 landmarks. For each case, the experiment is repeated 50 times and an uniformly distributed noise on the interval $[-0.5 ; 0.5]$ is added to the angular measurements. The initial covariance matrix $\left(P_{0}\right)$ for the EKF is set at $0.1 \mathrm{~m}$ on the diagonal for the position and 0rad on the diagonal for the orientation and 0 elsewhere.

a) First experimentation: EKF knows the true noise characteristics: Tab. II shows the results obtained by both algorithms. For each algorithm the mean error and the variance of error are given.

\begin{tabular}{|c|c|c|c|c|c|}
\hline number of landmarks & 1 & 5 & 10 & 50 & 100 \\
\hline$e_{A L I E N \text { in (m) }}$ & 0.0552 & 0.0263 & 0.0199 & 0.0120 & 0.0100 \\
$\sigma_{A L I E N \text { in (m) }}$ & 0.0031 & $5.1451 \mathrm{e}-004$ & $2.7911 \mathrm{e}-004$ & $7.9280 \mathrm{e}-005$ & $5.8512 \mathrm{e}-005$ \\
$e_{E K F}$ in (m) & 0.0897 & 0.0308 & 0.0224 & 0.0138 & 0.0116 \\
$\sigma_{E K F \text { en (m) }}$ & 0.0012 & $8.5777 \mathrm{e}-004$ & $5.0071 \mathrm{e}-004$ & $1.5034 \mathrm{e}-004$ & $9.6251 \mathrm{e}-005$ \\
$\frac{t_{A L I E N}}{t_{E K F}}$ & 0.6114 & 1.0971 & 1.2504 & 0.6544 & 0.7351 \\
\hline
\end{tabular}

TABLE II

COMPARATIVE RESULTS OF BOTH METHODS WHEN EKF DOES NOT KNOW THE NOISE CHARACTERISTICS

We can notice that there are no significant differences between the two algorithms in terms of mean estimation error and variance of estimation error, but the ALIEN algorithm computing time $\left(t_{A L I E N}\right)$ is really competitive with respect to the EKF one $\left(t_{E K F}\right)$ when the number of landmarks is below 4 or increases a lot.

Fig. 3 shows the results obtained for one run and for one landmark.

b) Second experimentation: EKF does not know the noise characteristics: Tab. III shows the results obtained by both algorithms. For each algorithm the mean estimation error and the variance estimation error are given.

\begin{tabular}{|c|c|c|c|c|c|}
\hline number of landmarks & 1 & 5 & 10 & 50 & 100 \\
\hline$e_{A L I E N}$ in (m) & 0.0456 & 0.0278 & 0.0216 & 0.0118 & 0.0100 \\
$\sigma_{A L I E N}$ & 0.0019 & $6.4213 \mathrm{e}-004$ & $3.3078 \mathrm{e}-004$ & $7.9920 \mathrm{e}-005$ & $5.6736 \mathrm{e}-005$ \\
$e_{E K F \text { in (m) }}$ & 0.1664 & 0.1595 & 0.1537 & 0.1178 & 0.0966 \\
$\sigma_{E K F \text { en (m) }}$ & $2.5201 \mathrm{e}-004$ & $1.4515 \mathrm{e}-004$ & $1.0377 \mathrm{e}-004$ & $8.6138 \mathrm{e}-004$ & 0.0017 \\
$\frac{t_{A L I E N}}{t_{E K F}}$ & 0.6214 & 1.0951 & 1.2487 & 0.7735 & 0.7371 \\
\hline
\end{tabular}

TABLE III

COMPARATIVE RESULTS OF BOTH METHODS WHEN EKF DOES NOT KNOW THE NOISE CHARACTERISTICS

EKF gives bad estimation results due to a bad initialisation which could not be compensated by the measurements because the algorithm has no knowledge about the noise characteristics. The ALIEN algorithm does not have this drawback because it does not need the noise characteristics in the estimation process. 

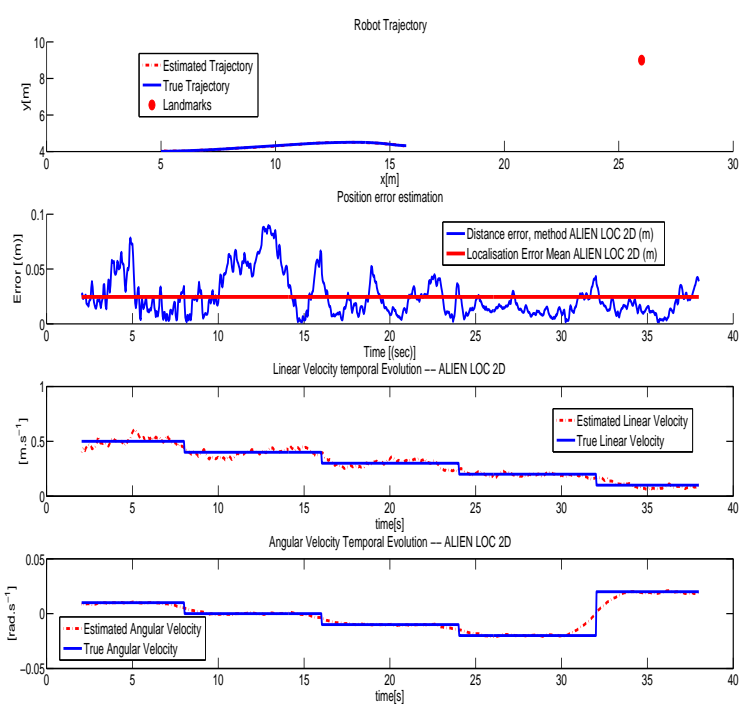

Fig. 3. Results for one run and for one landmark

\section{CONCLUSION}

This paper has proposed a new landmark based localization algorithm for a unicycle mobile robot. One of the most important advantage of this algorithm is that it is able to localize the robot with only one landmark. If the robot can only measure the relative angle between itself and the landmark in a 2D case, the proposed solution allows to localize the robot with respect to the landmark. In this case the control input (velocities: linear and angular ones) have to be known. Due to the drift problem in robotic navigation it can be useful to also estimate the linear and angular speed of the robot. This paper has proposed a solution to this problem by using only the relative angles between the robot and a single landmark in the 3D case. The theoretical development is implemented in Matlab in order to show the effectiveness of the proposed solution. Moreover, these results have been compared with those given by an Extended Kalman Filter, which is a reference in the mobile robotics community. A number of conclusions can be drawn from these experiments. First of all the more landmarks there are the more the ratio between the computing time necessary for the localization using the new algorithm and that using the EKF becomes favorable to the proposed algorithms.

The new proposed algorithm requires no knowledge of velocities (linear velocity and angular velocity), unlike the EKF which needs to know these inputs. Nevertheless, it is possible to extend the EKF to this case, but it requires a number of new theoretical developments, because there is no model of evolution for the velocities.

Moreover, for similar results in terms of error and variance, the paper proposes a new algorithm which is simpler to implement and more efficient in terms of computing time than the EKF one. However, the advantage of the EKF is its ability to give a confidence interval estimation. It is not yet possible for the proposed algorithm to give such a confidence interval, but it will the main subject of our future work. Concerning the initialization of the algorithm, it is not possible to conclude, because the EKF can be initialized with a least squares estimator using fifty measurements, in the same way as the ALIEN algorithm needs fifty measurements to give a first estimate of the position.

Another great advantage of the new algorithm is the lack of statistical assumptions on noise measurements, which makes it more robust with respect to any type of sensors. The future works concerns the study of the placement of the landmark and the choice of the landmarks in function of the current situation.

\section{REFERENCES}

[1] J. Borenstein, H. Everett, and L. Feng, "Where am I? Sensors and methods for mobile robot positioning," University of Michigan, 1996.

[2] J. Borenstein, "Control and kinematic design of multi-degree-of freedom mobile robots with compliant linkage," IEEE Transactions on Robotics and Automation, vol. 11, no. 1, pp. 21-35, 1995.

[3] F. Conticelli, A. Bicchi, and A. Balestrino, "Observability and nonlinear observers for mobile robot localization," in IFAC International Symposium on Robot Control, 2000.

[4] A. Bicchi, D. Prattichizzo, A. Marigo, and A. Balestrino, "On the observability of mobile vehicles localization," in IEEE Mediterranean Conference on Control and Systems, 1998.

[5] A. Martinelli and R. Siegwart, "Observability analysis for mobile robot localization," in IEEE/RSJ International Conference on Intelligent Robots and Systems, 2005, pp. 1471-1476.

[6] A. Bais, R. Sablatnig, and J. Gu, "Single landmark based selflocalization of mobile robots," Computer and Robot Vision, Canadian Conference, p. 67, 2006.

[7] G. Jang, S. Kim, J. Kim, and I. Kweon, "Metric localization using a single artificial landmark for indoor mobile robots," in IEEE International Conference on Intelligent Robots and Systems, 2005, pp. 1992 - 1997.

[8] T. Lemaire, S. Lacroix, and J. Sola, "A practical 3D bearing-only SLAM algorithm," in IEEE/RSJ International Conference on Intelligent Robots and Systems, 2005, pp. 2449-2454.

[9] N. Dao, B. You, and S. Oh, "Visual navigation for indoor mobile robots using a single camera," in IEEE International Conference on Intelligent Robots and Systems, 2005.

[10] S. Diop and M. Fliess, "Nonlinear observability, identifiability, and persistent trajectories," in Proceedings of the 30th IEEE Conference on Decision and Control, Brighton, England, December 1991.

[11] — , "On nonlinear observability," in Proc. 1st Europ. Control Conf, 1991, pp. 152-157.

[12] H. Leiva and S. Siegmund, "A necessary algebraic condition for controllability and observability of linear time-varying systems," IEEE Transactions on Automatic Control, vol. 48, no. 12, pp. 2229-2232, 2003.

[13] M. Mboup, C. Join, and M. Fliess, "Numerical differentiation with annihilators in noisy environment," Numerical Algorithms, vol. 50, pp. 439-467, 2009.

[14] M. Fliess and H. Sira-Ramírez, "An algebraic framework for linear identification," ESAIM Control Optim. Calc. Variat., vol. 9, pp. 151$168,2003$.

[15] H. Sorenson, Kalman filtering: theory and application. IEEE Press, 1985. 